\title{
HALF LIGHTLIKE SUBMANIFOLDS OF A SEMI-RIEMANNIAN SPACE FORM WITH A SEMI-SYMMETRIC NON-METRIC CONNECTION
}

\author{
DAE Ho JiN
}

\begin{abstract}
In this paper, we study screen quasi-conformal irrotational half lightlike submanifolds $M$ of a semi-Riemannian space form $\widetilde{M}(c)$ admitting a semi-symmetric non-metric connection, whose structure vector field $\zeta$ is tangent to $M$. The main result is a classification theorem for such Einstein half lightlike submanifolds of a Lorentzian space form admitting a semi-symmetric non-metric connection.
\end{abstract}

\section{INTRODUCTION}

The theory of lightlike submanifolds is indeed important for both the geometry of submanifolds to mathematics and its applications to physics. The study of such notion was initiated by Duggal and Bejancu [3] and later studied by many authors (see up-to date in $[4,5]$ ). The notion of a semi-symmetric non-metric connection on a Riemannian manifold was introduced by Ageshe and Chafle [1]. Although now we have lightlike version of a large variety of Riemannian submanifolds, the geometry of lightlike submanifolds of semi-Riemannian manifolds admitting semi-symmetric non-metric connections has been few known. Recently Yasar, Cöken and Yücesan $[15]$ and Jin $[6,7]$ studied lightlike hypersurfaces in a semi-Riemannian manifold admitting a semi-symmetric non-metric connection. Jin [10] and Jin-Lee [11] studied general lightlike submanifolds and half lightlike submanifolds of a semi-Riemannian manifold with a semi-symmetric non-metric connection.

The objective of this paper is to study screen quasi-conformal irrotational half lightlike submanifolds $M$ of a semi-Riemannian space form $\widetilde{M}(c)$ admitting a semisymmetric non-metric connection, whose structure vector field $\zeta$ of $\widetilde{M}(c)$ is tangent to $M$ but it does not belongs to $S(T M)$. The reason for this geometric restriction on

Received by the editors August 27, 2013. Accepted December 18, 2013

2010 Mathematics Subject Classification. 53C25, 53C40, 53C50.

Key words and phrases. screen quasi-conformal, half lightlike submanifold, semi-symmetric nonmetric connection. 
$M$ is due to the fact that such a class admits an integrable screen distribution and a symmetric induced Ricci tensor of $M$. Our main result is a classification theorem for such Einstein half lightlike submanifolds $M$ of a Lorentzian space form admitting a semi-symmetric non-metric connection.

\section{Semi-Symmetric Non-Metric Connection}

Let $(\widetilde{M}, \widetilde{g})$ be a semi-Riemannian manifold. A connection $\widetilde{\nabla}$ on $\widetilde{M}$ is called a semi-symmetric non-metric connection [1] if $\widetilde{\nabla}$ and its torsion tensor $\widetilde{T}$ satisfy

$$
\begin{gathered}
\left(\widetilde{\nabla}_{X} \widetilde{g}\right)(Y, Z)=-\pi(Y) \widetilde{g}(X, Z)-\pi(Z) \widetilde{g}(X, Y), \\
\widetilde{T}(X, Y)=\pi(Y) X-\pi(X) Y,
\end{gathered}
$$

for any vector fields $X, Y$ and $Z$ on $\widetilde{M}$, where $\pi$ is a 1 -form associated with a non-vanishing vector field $\zeta$, which is called the structure vector field, by

$$
\pi(X)=\widetilde{g}(X, \zeta)
$$

A submanifold $(M, g)$ of codimension 2 is called half lightlike submanifold if the radical distribution $\operatorname{Rad}(T M)=T M \cap T M^{\perp}$ is a vector subbundle of the tangent bundle $T M$ and the normal bundle $T M^{\perp}$ of $M$, with rank 1 . In this case, there exists complementary non-degenerate distributions $S(T M)$ and $S\left(T M^{\perp}\right)$ of $\operatorname{Rad}(T M)$ in $T M$ and $T M^{\perp}$ respectively, which are called the screen and co-screen distributions on $M$ respectively, such that

$$
T M=\operatorname{Rad}(T M) \oplus_{\text {orth }} S(T M), \quad T M^{\perp}=\operatorname{Rad}(T M) \oplus_{\text {orth }} S\left(T M^{\perp}\right),
$$

where $\oplus_{\text {orth }}$ denotes the orthogonal direct sum. We denote such a half lightlike submanifold by $M=(M, g, S(T M))$. Denote by $F(M)$ the algebra of smooth functions on $M$, by $\Gamma(E)$ the $F(M)$ module of smooth sections of a vector bundle $E$ over $M$ and by $(2.3)_{i}$ the $i$-th equation of (2.3). We use same notations for any others. Choose $L \in \Gamma\left(S\left(T M^{\perp}\right)\right)$ as a spacelike unit vector field, without loss of generality, i.e., $\widetilde{g}(L, L)=1$. We call $L$ the canonical normal vector field of $M$. Consider the orthogonal complementary vector bundle $S(T M)^{\perp}$ to $S(T M)$ in $T \widetilde{M}$. Certainly $\operatorname{Rad}(T M)$ and $S\left(T M^{\perp}\right)$ are vector subbundles of $S(T M)^{\perp}$. As $S\left(T M^{\perp}\right)$ is non-degenerate, we have

$$
S(T M)^{\perp}=S\left(T M^{\perp}\right) \oplus_{\text {orth }} S\left(T M^{\perp}\right)^{\perp},
$$

where $S\left(T M^{\perp}\right)^{\perp}$ is the orthogonal complementary to $S\left(T M^{\perp}\right)$ in $S(T M)^{\perp}$. It is well-known [3] that, for any null section $\xi$ of $\operatorname{Rad}(T M)$ on a coordinate neighborhood 
$\mathcal{U} \subset M$, there exists a uniquely defined lightlike vector bundle $\operatorname{lt}(T M)$ and a null vector field $N$ of $\operatorname{ltr}(T M)$ on $\mathcal{U}$ satisfying

$$
\widetilde{g}(\xi, N)=1, \widetilde{g}(N, N)=\widetilde{g}(N, X)=\widetilde{g}(N, L)=0, \forall X \in \Gamma(S(T M)) .
$$

We call $N, \operatorname{ltr}(T M)$ and $\operatorname{tr}(T M)=S\left(T M^{\perp}\right) \oplus_{\text {orth }}$ ltr $(T M)$ the lightlike transversal vector field, lightlike transversal vector bundle and transversal vector bundle of $M$ with respect to $S(T M)$ respectively [11]. Then $T \widetilde{M}$ is decomposed as

$$
\begin{aligned}
T \widetilde{M} & =T M \oplus \operatorname{tr}(T M)=\{\operatorname{Rad}(T M) \oplus \operatorname{tr}(T M)\} \oplus_{\text {orth }} S(T M) \\
& =\{\operatorname{Rad}(T M) \oplus l \operatorname{tr}(T M)\} \oplus_{\text {orth }} S(T M) \oplus_{\text {orth }} S\left(T M^{\perp}\right) .
\end{aligned}
$$

In the entire discussion of this article, we shall assume that the structure vector field $\zeta$ of $\widetilde{M}$ to be spacelike unit tangent vector field of $M$. In the sequel, we take $X, Y, Z, W \in \Gamma(T M)$, unless otherwise specified. Let $P$ be the projection morphism of $T M$ on $S(T M)$ with respect to the decomposition $(2.3)_{1}$. Then the local Gauss and Weingartan formulas of $M$ and $S(T M)$ are given respectively by

$$
\begin{aligned}
& \widetilde{\nabla}_{X} Y=\nabla_{X} Y+B(X, Y) N+D(X, Y) L, \\
& \widetilde{\nabla}_{X} N=-A_{N} X+\tau(X) N+\rho(X) L, \\
& \widetilde{\nabla}_{X} L=-A_{L} X+\phi(X) N ; \\
& \nabla_{X} P Y=\nabla_{X}^{*} P Y+C(X, P Y) \xi, \\
& \nabla_{X} \xi=-A_{\xi}^{*} X-\tau(X) \xi,
\end{aligned}
$$

where $\nabla$ and $\nabla^{*}$ are induced linear connections on $T M$ and $S(T M)$ respectively, $B$ and $D$ are called the local second fundamental forms of $M, C$ is called the local second fundamental form on $S(T M) . A_{N}, A_{\xi}^{*}$ and $A_{L}$ are linear operators on $T M$, which are called the shape operators, and $\tau, \rho$ and $\phi$ are 1-forms on $T M$. We say that $h(X, Y)=B(X, Y) N+D(X, Y) L$ is the global second fundamental form tensor of $M$. Using (2.1), (2.2) and (2.5), we have

$$
\begin{gathered}
\left(\nabla_{X} g\right)(Y, Z)=B(X, Y) \eta(Z)+B(X, Z) \eta(Y) \\
-\pi(Y) g(X, Z)-\pi(Z) g(X, Y) \\
T(X, Y)=\pi(Y) X-\pi(X) Y
\end{gathered}
$$

and $B$ and $D$ are symmetric on $T M$, where $T$ is the torsion tensor with respect to the induced connection $\nabla$ and $\eta$ is a 1 -form on $T M$ such that

$$
\eta(X)=\widetilde{g}(X, N) .
$$


From the facts $B(X, Y)=\widetilde{g}\left(\widetilde{\nabla}_{X} Y, \xi\right)$ and $D(X, Y)=\widetilde{g}\left(\widetilde{\nabla}_{X} Y, L\right)$, we know that $B$ and $D$ are independent of the choice of $S(T M)$ and satisfy

$$
B(X, \xi)=0, \quad D(X, \xi)=-\phi(X) .
$$

The above three local second fundamental forms $M$ and $S(T M)$ are related to their shape operators by

$$
\begin{aligned}
& g\left(A_{\xi}^{*} X, Y\right)=B(X, Y), \quad \widetilde{g}\left(A_{\xi}^{*} X, N\right)=0, \\
& g\left(A_{L} X, Y\right)=D(X, Y)+\phi(X) \eta(Y), \quad \widetilde{g}\left(A_{L} X, N\right)=\rho(X), \\
& g\left(A_{N} X, P Y\right)=C(X, P Y)-f g(X, P Y)-\eta(X) \pi(P Y), \\
& \widetilde{g}\left(A_{N} X, N\right)=-f \eta(X),
\end{aligned}
$$

where $f$ is the smooth function given by $f=\pi(N)$. From (2.12) and (2.13), we show that $A_{\xi}^{*}$ is $S(T M)$-valued self-adjoint and satisfies

$$
A_{\xi}^{*} \xi=0
$$

Denote by $\widetilde{R}, R$ and $R^{*}$ the curvature tensors of the semi-symmetric non-metric connection $\widetilde{\nabla}$ of $\widetilde{M}$, the induced connection $\nabla$ on $M$ and the induced connection $\nabla^{*}$ on $S(T M)$. Using the Gauss-Weingarten formulas for $M$ and $S(T M)$, we obtain the Gauss-Codazzi equations for $M$ and $S(T M)$ :

$$
\begin{aligned}
\widetilde{R}(X, Y) Z= & R(X, Y) Z+B(X, Z) A_{N} Y-B(Y, Z) A_{N} X \\
& +D(X, Z) A_{L} Y-D(Y, Z) A_{L} X \\
& +\left\{\left(\nabla_{X} B\right)(Y, Z)-\left(\nabla_{Y} B\right)(X, Z)\right. \\
& +B(Y, Z)[\tau(X)-\pi(X)]-B(X, Z)[\tau(Y)-\pi(Y)] \\
& +D(Y, Z) \phi(X)-D(X, Z) \phi(Y)\} N \\
& +\left\{\left(\nabla_{X} D\right)(Y, Z)-\left(\nabla_{Y} D\right)(X, Z)+B(Y, Z) \rho(X)\right. \\
& -B(X, Z) \rho(Y)-D(Y, Z) \pi(X)+D(X, Z) \pi(Y)\} L, \\
\widetilde{R}(X, Y) N= & -\nabla_{X}\left(A_{N} Y\right)+\nabla_{Y}\left(A_{N} X\right)+A_{N}[X, Y] \\
& +\tau(X) A_{N} Y-\tau(Y) A_{N} X+\rho(X) A_{L} Y-\rho(Y) A_{L} X \\
& +\left\{B\left(Y, A_{N} X\right)-B\left(X, A_{N} Y\right)+2 d \tau(X, Y)\right. \\
& +\phi(X) \rho(Y)-\phi(Y) \rho(X)\} N \\
& +\left\{D\left(Y, A_{N} X\right)-D\left(X, A_{N} Y\right)+2 d \rho(X, Y)\right. \\
& +\rho(X) \tau(Y)-\rho(Y) \tau(X)\} L,
\end{aligned}
$$




$$
\begin{aligned}
\widetilde{R}(X, Y) L=- & \nabla_{X}\left(A_{L} Y\right)+\nabla_{Y}\left(A_{L} X\right)+A_{L}[X, Y] \\
+ & \phi(X) A_{N} Y-\phi(Y) A_{N} X \\
+ & \left\{B\left(Y, A_{L} X\right)-B\left(X, A_{L} Y\right)+2 d \phi(X, Y)\right. \\
+ & \tau(X) \phi(Y)-\tau(Y) \phi(X)\} N \\
+ & \left\{D\left(Y, A_{L} X\right)-D\left(X, A_{L} Y\right)+\rho(X) \phi(Y)-\rho(Y) \phi(X)\right\} L ; \\
R(X, Y) P Z= & R^{*}(X, Y) P Z+C(X, P Z) A_{\xi}^{*} Y-C(Y, P Z) A_{\xi} X \\
& +\left\{\left(\nabla_{X} C\right)(Y, P Z)-\left(\nabla_{Y} C\right)(X, P Z)\right. \\
& +C(X, P Z)[\tau(Y)+\pi(Y)]-C(Y, P Z)[\tau(X)+\pi(X)]\} \xi \\
R(X, Y) \xi=- & \nabla_{X}^{*}\left(A_{\xi}^{*} Y\right)+\nabla_{Y}^{*}\left(A_{\xi}^{*} X\right)+A_{\xi}^{*}[X, Y] \\
& +\tau(Y) A_{\xi}^{*} X-\tau(X) A_{\xi}^{*} Y \\
& +\left\{C\left(Y, A_{\xi}^{*} X\right)-C\left(X, A_{\xi}^{*} Y\right)-2 d \tau(X, Y)\right. \\
& +\rho(X) \phi(Y)-\rho(Y) \phi(X)\} \xi .
\end{aligned}
$$

A complete simply connected semi-Riemannian manifold $\widetilde{M}$ of constant curvature $c$ is called a semi-Riemannian space form and denote it by $\widetilde{M}(c)$. For any $X, Y, Z \in$ $\Gamma(T \widetilde{M})$, the curvature tensor $\widetilde{R}$ of $\widetilde{M}(c)$ is given by

$$
\widetilde{R}(X, Y) Z=c\{\widetilde{g}(Y, Z) X-\widetilde{g}(X, Z) Y\} .
$$

Taking the scalar product with $\xi$ and $L$ to $(2.22)$, we get

$$
\widetilde{g}(\widetilde{R}(X, Y) Z, \xi)=\widetilde{g}(\widetilde{R}(X, Y) Z, L)=0, \quad \forall X, Y, Z \in \Gamma(T M) .
$$

From this results and (2.17), for all $X, Y, Z \in \Gamma(T M)$, we obtain

$$
\begin{gathered}
\widetilde{R}(X, Y) Z=R(X, Y) Z+B(X, Z) A_{N} Y-B(Y, Z) A_{N} X \\
+D(X, Z) A_{L} Y-D(Y, Z) A_{L} X .
\end{gathered}
$$

\section{Characterization Theorems}

Definition. A half lightlike submanifold $M$ of a semi-Riemannian manifold $\widetilde{M}$ is said to be irrotational [12] if $\widetilde{\nabla}_{X} \xi \in \Gamma(T M)$ for any $X \in \Gamma(T M)$.

From (2.5) and (2.12), we show that the above definition is equivalent to the condition: $D(X, \xi)=0=\phi(X)$ for all $X \in \Gamma(T M)$.

Lemma 1 ([8, 11]). Let $M$ be an irrotational half lightlike submanifold of a semiRiemannian manifold $\widetilde{M}$ admitting a semi-symmetric non-metric connection such 
that the structure vector field $\zeta$ of $\widetilde{M}$ is tangent to $M$. Then $\zeta$ is conjugate to any vector field $X$ on $M$, i.e., $\zeta$ satisfies $h(X, \zeta)=0$.

Note that $h(X, \zeta)=0$ is equivalent to the following two equations:

$$
B(X, \zeta)=\pi\left(A_{\xi}^{*} X\right)=0, \quad D(X, \zeta)=\pi\left(A_{L} X\right)=0, \quad \forall X \in \Gamma(T M) .
$$

Definition. A half lightlike submanifold $M$ of a semi-Riemannian manifold $\widetilde{M}$ admitting a semi-symmetric non-metric connection is called screen quasi-conformal $[9,13]$ if the second fundamental forms $B$ and $C$ satisfy

$$
C(X, P Y)=\varphi B(X, Y)+\eta(X) \pi(P Y),
$$

where $\varphi$ is a non-vanishing function on a coordinate neighborhood $\mathcal{U}$ in $M$.

Due to (2.13) and (2.15), we show that $M$ is screen quasi-conformal if and only if the shape operators $A_{N}$ and $A_{\xi}^{*}$ are related by

$$
A_{N} X=\varphi A_{\xi}^{*} X-f X .
$$

We quote the following results for irrotational screen quasi-conformal half lightlike submanifold due to Jin [9]:

Theorem 3.1. Let $M$ be an irrotational screen quasi-conformal half lightlike submanifolds $M$ of a semi-Riemannian space form $\widetilde{M}(c)$ admitting a semi-symmetric non-metric connection. If the structure vector field $\zeta$ is tangent to $M$ but it does not belong to $S(T M)$, then we have $c=1$.

Let $\widetilde{R i c}$ be the Ricci curvature tensor of $\widetilde{M}$ and $R^{(0,2)}$ the induced Ricci type tensor on $M$ given respectively by

$$
\begin{aligned}
& \widetilde{\operatorname{Ric}}(X, Y)=\operatorname{trace}\{Z \rightarrow \widetilde{R}(Z, X) Y\}, \quad \forall X, Y \in \Gamma(T \widetilde{M}), \\
& R^{(0,2)}(X, Y)=\operatorname{trace}\{Z \rightarrow R(Z, X) Y\}, \quad \forall X, Y \in \Gamma(T M) .
\end{aligned}
$$

Consider a quasi-orthonormal frame field $\left\{\xi ; W_{a}\right\}$ on $M$, where $\operatorname{Rad}(T M)=$ $\operatorname{Span}\{\xi\}$ and $S(T M)=\operatorname{Span}\left\{W_{a}\right\}$ and let $E=\left\{\xi, N, W_{a}\right\}$ be the corresponding frame field on $\widetilde{M}$. Using this quasi-orthonormal frame field, we obtain

$$
\begin{aligned}
R^{(0,2)}(X, Y)= & \widetilde{\operatorname{Ric}}(X, Y)+B(X, Y) \operatorname{tr} A_{N}+D(X, Y) \operatorname{tr} A_{L} \\
& -g\left(A_{N} X, A_{\xi}^{*} Y\right)-g\left(A_{L} X, A_{L} Y\right)+\rho(X) \phi(Y) \\
& -\widetilde{g}(\widetilde{R}(\xi, Y) X, N)-\widetilde{g}(\widetilde{R}(L, X) Y, L),
\end{aligned}
$$

This shows that $R^{(0,2)}$ is not symmetric. The tensor field $R^{(0,2)}$ is called its induced Ricci tensor [4, 5], denoted by Ric, of $M$ if it is symmetric. It is known [11] that 
$R^{(0,2)}$ is an induced Ricci tensor of $M$ if and only if the 1-form $\tau$ is closed, i.e., $d \tau=0$, for any coordinate neighborhood $\mathcal{U} \subset M$.

Remark 1. If $R^{(0,2)}$ is symmetric, then there exists a null pair $\{\xi, N\}$ such that the corresponding 1-form $\tau$ satisfies $\tau=0$ [11], which called a canonical null pair of $M$. Although $S(T M)$ is not unique, it is canonically isomorphic to the factor vector bundle $S(T M)^{\sharp}=T M / \operatorname{Rad}(T M)$ [12]. This implies that all screen distribution are mutually isomorphic. For this reason, in case $d \tau=0$ we consider only lightlike hypersurfaces $M$ endow with the canonical null pair.

We say that $M$ is an Einstein manifold if the Ricci tensor of $M$ satisfies

$$
R i c=\kappa g .
$$

It is well-known that if $\operatorname{dim} M>2$, then $\kappa$ is a constant. For $\operatorname{dim} M=2$, any manifold $M$ is Einstein but $\kappa$ is not necessarily constant.

In case the ambient manifold $\widetilde{M}$ is a space form $\widetilde{M}(c), R^{(0,2)}$ is given by

$$
\begin{aligned}
R^{(0,2)}(X, Y) & =m c g(X, Y)+B(X, Y) \operatorname{tr} A_{N}+D(X, Y) \operatorname{tr} A_{L} \\
& -g\left(A_{N} X, A_{\xi}^{*} Y\right)-g\left(A_{L} X, A_{L} Y\right)+\rho(X) \phi(Y) .
\end{aligned}
$$

Taking the scalar product with $\xi$ to (2.17) and using (2.22), we have

$$
\begin{aligned}
& \left(\nabla_{X} B\right)(Y, Z)-\left(\nabla_{Y} B\right)(X, Z) \\
& =B(Y, Z)\{\pi(X)-\tau(X)\}-B(X, Z)\{\pi(Y)-\tau(Y)\} .
\end{aligned}
$$

Definition. A vector field $X$ on $\widetilde{M}$ is said to be conformal Killing [8] if

$$
\widetilde{\mathcal{L}}_{X} \widetilde{g}=-2 \delta \widetilde{g}
$$

for any non-vanishing smooth function $\delta$, where $\widetilde{\mathcal{L}}$ denotes the Lie derivative on $\widetilde{M}$, that is, for all $Y, Z \in \Gamma(T \widetilde{M})$,

$$
\left(\widetilde{\mathcal{L}}_{X} \widetilde{g}\right)(Y, Z)=X(\widetilde{g}(Y, Z))-\widetilde{g}([X, Y], Z)-\widetilde{g}(Y,[X, Z]) .
$$

In particular, if $\delta=0$, then $X$ is called a Killing vector field on $\widetilde{M}$.

Theorem 3.2 ([8, 11]). Let $M$ be a half lightlike submanifold of $\widetilde{M}$ admitting a semi-symmetric non-metric connection. If the canonical normal vector field $L$ is conformal Killing, then $L$ is a Killing vector field.

Proof. Using (2.1) and (2.2), for any $X, Y, Z \in \Gamma(T \widetilde{M})$, we have

$$
\left(\widetilde{\mathcal{L}}_{X} \widetilde{g}\right)(Y, Z)=\widetilde{g}\left(\widetilde{\nabla}_{Y} X, Z\right)+\widetilde{g}\left(Y, \widetilde{\nabla}_{Z} X\right)-2 \pi(X) \widetilde{g}(Y, Z) .
$$


As $L$ is conformal Killing, we have $\widetilde{g}\left(\widetilde{\nabla}_{X} L, Y\right)=-D(X, Y)$ by (2.9) and (2.16). This implies $\left(\widetilde{\mathcal{L}}_{L} \widetilde{g}\right)(X, Y)=-2 D(X, Y)$ for any $X, Y \in \Gamma(T M)$. Thus we have

$$
D(X, Y)=\delta g(X, Y), \quad \forall X, Y \in \Gamma(T M) .
$$

Taking $X=Y=\zeta$ to this and using $(3.1)_{2}$, we get $\delta=0$ and $L$ is Killing.

Theorem 3.3 ([11]). Let $M$ be a half lightlike submanifold of a semi-Riemannian manifold $\widetilde{M}$ admitting a semi-symmetric metric connection. Then the following assertions are equivalent:

(1) The screen distribution $S(T M)$ is an integrable distribution.

(2) $C$ is symmetric, i.e., $C(X, Y)=C(Y, X)$ for all $X, Y \in \Gamma(S(T M))$.

(3) The shape operator $A_{N}$ is self-adjoint with respect to $g$, i.e.,

$$
g\left(A_{N} X, Y\right)=g\left(X, A_{N} Y\right), \quad \forall X, Y \in \Gamma(S(T M)) .
$$

Remark 2. Just as in the well-known case of locally product Riemannian or semiRiemannian manifolds [3, 4, 5, 14], if $S(T M)$ is an integrable distribution, then $M$ is locally a product manifold $\mathcal{C} \times M^{*}$ where $\mathcal{C}$ is a null curve tangent to $\operatorname{Rad}(T M)$ and $M^{*}$ is a leaf of the integrable distribution $S(T M)$.

Theorem 3.4. Let $M$ be a screen quasi-conformal irrotational Einstein half lightlike submanifold of a Lorentzian space form $\widetilde{M}(c)$ with a semi-symmetric non-metric connection. If $\zeta$ is tangent to $M$ but it does not belong to $S(T M)$, the canonical normal vector field is conformal Killing and the mean curvature of $M$ is constant, then $M$ is locally a product manifold $M=\mathcal{C} \times M_{1} \times M_{2}$, where $\mathcal{C}$ is a null curve, $M_{1}$ is an Euclidean space and $M_{2}$ is a totally umbilical Riemannian space.

Proof. As $L$ is Killing, we get $D=\phi=0$ and $g\left(A_{L} X, Y\right)=0$ for any $X, Y \in$ $\Gamma(T M)$. From $(3.3),(3.5)$ and the fact $A_{\xi}^{*}$ is self-adjoint, we show that $R^{(0,2)}$ is a symmetric induced Ricci tensor Ric and $S(T M)$ is an integrable distribution. As $g\left(A_{\xi}^{*} \zeta, X\right)=B(\zeta, X)=0$ and $S(T M)$ is non-degenerate, we have

$$
A_{\xi}^{*} \zeta=0
$$

Using (2.13), (3.3), (3.4) and the fact $c=1$, from (3.5) we have

$$
g\left(A_{\xi}^{*} X, A_{\xi}^{*} Y\right)-\alpha g\left(A_{\xi}^{*} X, Y\right)+\varphi^{-1}(\kappa-m) g(X, Y)=0,
$$

for all $X, Y \in \Gamma(T M)$ due to $c=1$, where $\alpha=\operatorname{tr} A_{\xi}^{*}-f m \varphi^{-1}$. Taking $X=Y=\zeta$ to (3.8) and using (3.7), we have $\kappa=m$. Thus (3.8) becomes

$$
g\left(A_{\xi}^{*} X, A_{\xi}^{*} Y\right)-\alpha g\left(A_{\xi}^{*} X, Y\right)=0 .
$$


As $\widetilde{M}$ is Lorentzian manifold, $S(T M)$ is a Riemannian. Since $\xi$ is an eigenvector field of $A_{\xi}^{*}$ corresponding to the eigenvalue 0 due to (2.16) and $A_{\xi}^{*}$ is $S(T M)$-valued real self-adjoint operator, $A_{\xi}^{*}$ have $m$ real orthonormal eigenvector fields in $S(T M)$ and is diagonalizable. Consider a frame field of eigenvectors $\left\{\xi, E_{1}, \ldots, E_{m}\right\}$ of $A_{\xi}^{*}$ such that $\left\{E_{1}, \ldots, E_{m}\right\}$ is an orthonormal frame field of $S(T M)$ and $A_{\xi}^{*} E_{i}=\lambda_{i} E_{i}$. Put $X=Y=E_{i}$ in (3.9), each eigenvalue $\lambda_{i}$ is a solution of the equation

$$
x^{2}-\alpha x=0 .
$$

As this equation has at most two distinct solutions 0 and $\alpha$, there exists $p \in$ $\{0,1, \ldots, m\}$ such that $\lambda_{1}=\cdots=\lambda_{p}=0$ and $\lambda_{p+1}=\cdots=\lambda_{m}=\alpha(\neq 0)$, by renumbering if necessary. As $\operatorname{tr} A_{\xi}^{*}=0 p+(m-p) \alpha$, we have

$$
(m-p-1) \alpha=f m \varphi^{-1} .
$$

Consider four distributions $D_{o}, D_{\alpha}, D_{o}^{s}$ and $D_{\alpha}^{s}$ on $S(T M)$ given by

$$
\begin{array}{ll}
D_{o}=\left\{X \in \Gamma(T M) \mid A_{\xi}^{*} X=0\right\}, & D_{o}^{s}=D_{o} \cap S(T M), \\
D_{\alpha}=\left\{U \in \Gamma(T M) \mid A_{\xi}^{*} U=\alpha P U\right\}, & D_{\alpha}^{s}=D_{\alpha} \cap S(T M) .
\end{array}
$$

Clearly we show that $D_{o} \cap D_{\alpha}=\operatorname{Rad}(T M), D_{o}^{s} \cap D_{\alpha}^{s}=\{0\}$ as $\alpha \neq 0$ and $D_{o}^{s}=$ $P D_{o}, D_{\alpha}^{s}=D_{\alpha}$. In the sequel, we take the vector fields $X, Y \in \Gamma\left(D_{o}\right), U, V \in$ $\Gamma\left(D_{\alpha}\right)$ and $Z, W \in \Gamma(T M)$. Denote $X^{*}=P X, Y^{*}=P Y, U^{*}=P U$ and $V^{*}=P V$. Then $X^{*}, Y^{*} \in \Gamma\left(D_{o}^{s}\right)$ and $U^{*}, V^{*} \in \Gamma\left(D_{\alpha}^{s}\right)$. Since $X^{*}$ and $U^{*}$ are eigenvector fields of the real self-adjoint operator $A_{\xi}^{*}$ corresponding to the different eigenvalues 0 and $\alpha$ respectively, $X^{*} \perp U^{*}$ and $g(X, U)=g\left(X^{*}, U^{*}\right)=0$, that is, $D_{o} \perp_{g} D_{\alpha}$. Also, since $B(X, U)=g\left(A_{\xi}^{*} X, U\right)=0$, we show that $D_{\alpha} \perp_{B} D_{o}$. Since $\left\{E_{i}\right\}_{1 \leq i \leq p}$ and $\left\{E_{a}\right\}_{p+1 \leq a \leq m}$ are vector fields of $D_{o}^{s}$ and $D_{\alpha}^{s}$ respectively and $D_{o}^{s}$ and $D_{\alpha}^{s}$ are mutually orthogonal, $D_{o}^{s}$ and $D_{\alpha}^{s}$ are non-degenerate distributions of rank $p$ and rank $(m-p)$ respectively. Thus $S(T M)$ is decomposed as $S(T M)=D_{\alpha}^{s} \oplus_{\text {orth }} D_{o}^{s}$.

From (3.9), we get $A_{\xi}^{*}\left(A_{\alpha}^{*}-\alpha P\right)=0$. Let $W \in \operatorname{Im} A_{\xi}^{*}$. Then there exists $Z \in \Gamma(T M)$ such that $W=A_{\xi}^{*} Z$. Then $\left(A_{\xi}^{*}-\alpha P\right) W=0$ and $W \in \Gamma\left(D_{\alpha}\right)$. Thus $\operatorname{Im} A_{\xi}^{*} \subset \Gamma\left(D_{\alpha}\right)$. By duality, we have $\operatorname{Im}\left(A_{\xi}^{*}-\alpha P\right) \subset \Gamma\left(D_{o}\right)$.

Applying $\nabla_{X}$ to $B(Y, U)=0$ and using $(2.13)$ and $A_{\xi}^{*} Y=0$, we obtain

$$
\left(\nabla_{X} B\right)(Y, U)=-g\left(A_{\xi}^{*} \nabla_{X} Y, U\right) .
$$

Substituting this into (3.6) and using (2.11) and $A_{\xi}^{*} X=A_{\xi}^{*} Y=0$, we get

$$
g\left(A_{\xi}^{*}[X, Y], U\right)=0 .
$$


As $\operatorname{Im} A_{\xi}^{*} \subset \Gamma\left(D_{\alpha}\right)$ and $D_{\alpha}$ is non-degenerate, we get $A_{\xi}^{*}[X, Y]=0$. Thus $[X, Y] \in$ $\Gamma\left(D_{o}\right)$ and $D_{o}$ is integrable. This result implies $\left[X^{*}, Y^{*}\right] \in \Gamma\left(D_{o}\right)$. On the other hand, since $S(T M)$ is integrable, $\left[X^{*}, Y^{*}\right] \in \Gamma(S(T M))$. Thus $\left[X^{*}, Y^{*}\right] \in \Gamma\left(D_{o}^{s}\right)$. Thus $D_{o}^{s}$ is also an integrable distribution.

Applying $\nabla_{V}$ to $B(U, Y)=0$ and using $A_{\xi}^{*} Y=0$ and $A_{\xi}^{*} U=\alpha P U$, we get

$$
\left(\nabla_{V} B\right)(U, Y)=-\alpha g\left(\nabla_{V} Y, U\right) .
$$

Substituting this into (3.6) and using the fact $\alpha \neq 0$, we obtain

$$
g\left(\nabla_{V} Y, U\right)=g\left(V, \nabla_{U} Y\right) .
$$

Applying $\nabla_{V}$ to $g(Y, U)=0$ and using (2.10), we have

$$
\pi(Y) g(U, V)-B(V, U) \eta(Y)-g\left(\nabla_{V} Y, U\right)=g\left(Y, \nabla_{V} U\right) .
$$

Taking the skew-symmetric part of this equation and using (2.11), we have

$$
g([V, U], Y)=0, \quad \forall Y \in \Gamma\left(D_{o}\right) \text { and } U, V \in \Gamma\left(D_{\alpha}\right) .
$$

From this, we get $g\left(\left[V^{*}, U^{*}\right], Y^{*}\right)=0$ for all $Y^{*} \in \Gamma\left(D_{o}^{s}\right)$ and $U^{*}, V^{*} \in \Gamma\left(D_{\alpha}^{s}\right)$. As $D_{o}^{s}$ and $D_{\alpha}^{s}$ are mutually orthogonal non-degenerate distributions, we show that $\left[V^{*}, U^{*}\right] \in \Gamma\left(D_{\alpha}^{S}\right)$. Thus $D_{\alpha}^{s}$ is also an integrable distribution.

Applying $\nabla_{U}$ to $B(X, Y)=0$ and $\nabla_{X}$ to $B(U, Y)=0$, we have

$$
\left(\nabla_{U} B\right)(X, Y)=0, \quad\left(\nabla_{X} B\right)(U, Y)=-\alpha g\left(\nabla_{X} Y, U\right) .
$$

Substituting this two equations into (3.6), we have $\alpha g\left(\nabla_{X} Y, U\right)=0$. As

$$
g\left(A_{\xi}^{*} \nabla_{X} Y, U\right)=B\left(\nabla_{X} Y, U\right)=\alpha g\left(\nabla_{X} Y, U\right)=0
$$

and $\operatorname{Im} A_{\xi}^{*} \subset \Gamma\left(D_{\alpha}\right)$ and $D_{\alpha}$ is non-degenerate, we get $A_{\xi}^{*} \nabla_{X} Y=0$. This implies $\nabla_{X} Y \in \Gamma\left(D_{o}\right)$. Thus $D_{o}$ is an auto-parallel distribution on $S(T M)$. This implies that $\nabla_{X^{*}} Y^{*} \in \Gamma\left(D_{o}\right)$ for any $X^{*}, Y^{*} \in \Gamma\left(D_{o}^{s}\right)$. As $C\left(X^{*}, Y^{*}\right)=\varphi B\left(X^{*}, Y^{*}\right)+$ $\eta\left(X^{*}\right) \pi\left(Y^{*}\right)=0$, we have $\nabla_{X^{*}} Y^{*}=\nabla_{X^{*}}^{*} Y^{*} \in \Gamma(S(T M))$. Thus $\nabla_{X^{*}} Y^{*} \in \Gamma\left(D_{o}^{s}\right)$ and $D_{o}^{s}$ is also an auto-parallel distribution.

As $A_{\xi}^{*} \zeta=0, \zeta$ belongs to $D_{o}$. Thus $\pi(U)=0$ for any $U \in \Gamma\left(D_{\alpha}\right)$. Applying $\nabla_{X}$ to $g(U, Y)=0$ and using (2.10) and the fact $D_{o}$ is auto-parallel, we get $g\left(\nabla_{X} U, Y\right)=0$. This implies $\nabla_{X} U \in \Gamma\left(D_{\alpha}\right)$.

Assume that the mean curvature vector field

$$
\mu=\frac{1}{m} g\left(A_{\xi}^{*} E_{a}, E_{a}\right)=\frac{m-p}{m} \alpha
$$


of $M$ is constant. Then $\alpha$ is a constant. Applying $\nabla_{X}$ to $B(U, V)=\alpha g(U, V)$ and $\nabla_{U}$ to $B(X, V)=0$ and using the fact $\alpha$ is constant, we have

$$
\left(\nabla_{X} B\right)(U, V)=0, \quad\left(\nabla_{U} B\right)(X, V)=-\alpha g\left(\nabla_{U} X, V\right) .
$$

Substituting this two equations into (3.6) and using $D_{o} \perp_{B} D_{\alpha}$, we have

$$
g\left(\nabla_{U} X, V\right)=\pi(X) g(U, V) .
$$

Applying $\nabla_{U}$ to $g(X, V)=0$ and using (2.10), we obtain

$$
g\left(X, \nabla_{U} V\right)=0 .
$$

From this, we get $g\left(X^{*}, \nabla_{U^{*}} V^{*}\right)=0$ for all $X^{*} \in \Gamma\left(D_{o}^{s}\right)$ and $U^{*}, V^{*} \in \Gamma\left(D_{\alpha}^{s}\right)$. As $D_{o}^{s}$ and $D_{\alpha}^{s}$ are mutually orthogonal non-degenerate distributions, we show that $\nabla_{U^{*}} V^{*} \in \Gamma\left(D_{\alpha}^{S}\right)$. Thus $D_{\alpha}^{s}$ is auto-parallel distribution.

Since the leaf $M^{*}$ of $S(T M)$ is a Riemannian manifold and $S(T M)=D_{\alpha}^{s} \oplus_{\text {orth }}$ $D_{o}^{s}$, where $D_{\alpha}^{s}$ and $D_{o}^{s}$ are auto-parallel distributions of $M^{*}$, by the decomposition theorem of de Rham [2] we have $M^{*}=M_{1} \times M_{2}$, where $M_{1}$ is a totally geodesic leaf of $D_{o}^{s}$ and $M_{2}$ is a totally umbilical leaf of $D_{\alpha}^{s}$. Consider the frame field of eigenvectors $\left\{\xi, E_{1}, \ldots, E_{m}\right\}$ of $A_{\xi}^{*}$ such that $\left\{E_{i}\right\}_{i}$ is an orthonormal frame field of $S(T M)$, then $B\left(E_{i}, E_{j}\right)=C\left(E_{i}, E_{j}\right)=0$ for $1 \leq i<j \leq m$ and $B\left(E_{i}, E_{i}\right)=C\left(E_{i}, E_{i}\right)=$ 0 for $1 \leq i \leq m-1$. From (2.17) and (2.20), we have $\widetilde{g}\left(\widetilde{R}\left(E_{i}, E_{j}\right) E_{j}, E_{i}\right)=$ $g\left(R^{*}\left(E_{i}, E_{j}\right) E_{j}, E_{i}\right)=0$. Thus the sectional curvature $K$ of $M_{2}$ is given by

$$
K\left(E_{i}, E_{j}\right)=\frac{g\left(R^{*}\left(E_{i}, E_{j}\right) E_{j}, E_{i}\right)}{g\left(E_{i}, E_{i}\right) g\left(E_{j}, E_{j}\right)-g^{2}\left(E_{i}, E_{j}\right)}=0 .
$$

Thus $M$ is a locally product $\mathcal{C} \times M_{1} \times M_{2}$, where $\mathcal{C}$ is a null curve, $M_{1}$ is an Euclidean space and $M_{2}$ is a totally umbilical Riemannian space.

\section{REFERENCES}

1. Ageshe, N.S. \& Chafle, M.R.: A semi-symmetric non-metric connection on a Riemannian manifold. Indian J. Pure Appl. Math. 23 (1992), no. 6, 399-409.

2. de Rham, G.: Sur la réductibilité d'un espace de Riemannian. Comm. Math. Helv. 26 (1952), 328-344.

3. Duggal, K.L. \& Bejancu, A.: Lightlike Submanifolds of Semi-Riemannian Manifolds and Applications. Kluwer Acad. Publishers, Dordrecht, 1996.

4. Duggal, K.L. \& Jin, D.H.: Null curves and Hypersurfaces of Semi-Riemannian Manifolds. World Scientific, 2007. 
5. Duggal, K.L. \& Sahin, B.: Differential geometry of lightlike submanifolds. Frontiers in Mathematics, Birkhäuser, 2010.

6. Jin, D.H.: Geometry of lightlike hypersurfaces of a semi-Riemannian space form with a semi-symmetric non-metric connection. Submitted in Indian J. Pure Appl. Math.

7. ___ Einstein lightlike hypersurfaces of a Lorentz space form with a semi-symmetric non-metric connection. Accepted in Bull. Korean Math. Soc. (2013).

8. ___ : Einstein half lightlike submanifolds of a Lorentzian space form with a semisymmetric non-metric connection. Submitted in Journal of Inequalities and Applications.

9. ___ Two characterization theorems for irrotational lightlike geometry. accepted in Comm. Korean Math. Soc. (2013).

10. ___ Lightlike submanifolds of a semi-Riemannian manifold with a semi-symmetric non-metric connection. J. Korean Soc Math. Edu. Ser. B: Pure Appl. Math. 19 (2012), no. $3,211-228$.

11. Jin, D.H \& Lee, J.W.: A classification of half lightlike submanifolds of a semiRiemannian manifold with a semi-symmetric non-metric connection. Bull. Korean Math. Soc. 50 (2013), no. 3, 705-717.

12. Kupeli, D.N.: Singular Semi-Riemannian Geometry. Kluwer Academic, 366, 1996.

13. Massamba, F.: Screen almost conformal lightlike geometry in indefinite Kenmotsu space forms. Int. Electron. J. Geom. 5 (2012), no. 2, 36-58.

14. O'Neill, B.: Semi-Riemannian Geometry with Applications to Relativity. Academic Press, 1983.

15. Yasar, E.; Cöken, A.C. \& Yücesan, A.: Lightlike hypersurfaces in semi-Riemannian manifold with semi-symmetric non-metric connection. Math. Scand. 102 (2008), 253264.

Department of Mathematics, Dongguk University, Gyeongue 780-714, Republic of KoREA

Email address: jindh@dongguk.ac.kr 